\title{
MEJORAMIENTO DEL SISTEMA DE CAPTACIÓN - ADUCCIÓN - DESARENADOR ACUEDUCTO MUNICIPIO DE CALARCÁ, QUINDÍO
}

\author{
IMPROVEMENT OF INTAKE WORK-MAIN TRANSMISSION LINE-SAND TRAP SYSTEM \\ IN AQUEDUCT OF CALARCA, QUINDIO
}

\author{
Elkin Aníbal Monsalve Durango ${ }^{1}$ \\ Pedro León García Reinoso ${ }^{1}$, Gabriel Lozano Sandoval ${ }^{1}$ \\ ${ }^{1}$ Programa de Ingeniería Civil. Facultad de Ingeniería. Universidad del Quindío. Investigadores Asociados Grupo CIDERA. \\ Fecha de recibido: Febrero 3 de 2010 \\ Fecha de aceptado: Junio 9 de 2010 \\ Correspondencia: Programa de Ingeniería Civil, Universidad del Quindío. Av. Bolivar calle 12 norte Armenia Quindío. Correo electrónico: \\ pedrogracia@uniquindio.edu.co
}

\section{RESUMEN}

La zona de capación del sistema de acueducto del Municipio de Calarcá en el Departamento del Quindío, localizada en la parte alta del Río Santo Domingo, constantemente quedaba inhabilitada por el alto flujo de material presente en este río de montaña con régimen torrencial. La empresa MULTIPROPÓSITO DE CALARCÁ S.A ESP encargada de prestar entre otros servicios el Acueducto del municipio, solicitó a la Universidad del Quindío, a través del Centro de Extensión de la Facultad de Ingeniería y con el apoyo del grupo de Investigación CIDERA, analizar la situación hidrológica e hidráulica presente, y realizar los estudios que permitieron establecer las acciones de intervención en la zona de captación y la optimización de cada una de las unidades y elementos conexos en captación, canal de aducción y desarenador.

Palabras Clave: Sistema de acueducto, bocatoma, canal de aducción.

\begin{abstract}
The water intake area of the aqueduct system in the town of Calarcá (Department of Quindio), located in the uppermost zone of Santo Domingo river, was constantly out of service due to the high flow of solid material carried by this mountain river, which is characterized by a turbulent flow regime. The Company MULTIPROPOSITO DE CALARCA S.A. ESP which is in charge of providing, among other services, the aqueduct service in the town, requested University of Quindio, through Extension Office of Faculty of Engineering and with support of research group CIDERA, to analyze the current hydrologic and hydraulic situation, and perform the studies that allow to determine the actions to be taken in the intake area and the upgrading of every unit and related elements in intake work, main transmission line and sand trap.
\end{abstract}

Key Words: Aqueduct System, Intake Structure, Main Transmission Line.

\section{INTRODUCCIÓN}

La EMPRESA MULTIPROPÓSITO DE CALARCÁ S.A. ESP abastece de agua al municipio de Calarcá a partir de 4 fuentes (Quebrada El Naranjal, San Rafael, El Salado y Río Santo Domingo) con una concesión total para uso doméstico de $220 \mathrm{l} / \mathrm{s}$. Las bocatomas integran los sistemas San Rafael, Naranjal y Santo Domingo Salado, ubicándose sobre diferentes microcuencas en la vertiente occidental de la cordillera Central, con un relieve muy escarpado (pendientes superiores al 50\%).
El río Santo Domingo donde se encuentra la principal captación de Calarcá, presenta un régimen torrencial, con tendencia a la generación de amplios caudales con fuerza de arrastre que logra desplazar grandes bloques y obstruía e inhabilitaba la obra de captación existente. El sistema de captación Santo Domingo - Salado no disponía de elementos para el control del caudal captado, lo que implicaba constantes reboses en el desarenador, impidiendo el funcionamiento óptimo de la unidad y generando bajas eficiencias en la 
remoción de material suspendido y lecturas por fuera del rango, respecto del caudal derivado a la planta de tratamiento.

En la zona de captación del río Santo Domingo hay constante presencia del material generado por el tránsito de crecientes, lo que ocasionó que en el pasado se presentara socavación y alteración de la estructura de captación, dando paso a volúmenes mayores a los requeridos. La aducción presentaba vertederos controlados fuera de operación, y la evacuación de excesos requería de operación manual, respondiendo a ajustes del operador y no a la dinámica de la corriente. La entrega de la aducción el Salado al canal principal, y la entrega de éste al desarenador, presentaba problemas hidráulicos que impedían el adecuado control de la velocidad.

Siendo las estructuras de captación elementos importantes para el abastecimiento de agua de una población, deben proyectarse de acuerdo con las condiciones geológicas y topográficas del lugar donde se realiza la extracción y a las variaciones del caudal a extraer; de igual manera, dependen naturalmente del tipo de uso y de las características hidrológicas e hidráulicas de la corriente de agua que se quiere aprovechar.

Los diversos sistemas de captación de agua constituidos en zonas de montaña, funcionan bajo condiciones y características muy particulares, por las altas pendientes de los cauces y los constantes flujos de material, dejando por fuera criterios y parámetros técnicos propios de los pocos textos académicos que ilustran sobre el diseño de acueductos en Colombia.

Como los diseños deben abordarse desde el entendimiento claro del problema, la realidad hidrológica de la cuenca y el comportamiento hidráulico en el tramo donde se desea ubicar la captación, hasta adaptarse así a los parámetros y criterios técnicos a los que hace referencia la literatura; la Universidad del Quindío, a través del Centro de Extensión de la Facultad de Ingeniería y con el apoyo del grupo de Investigación CIDERA, se propuso mediante una visión holística conocer y analizar las variables presentes en estas zonas de captación de alta montaña, integrando la particularidad que hay en cuanto a las condiciones hidrológicas e hidráulicas y los criterios de diseño de acueductos que reporta la literatura, hasta documentar detalladamente el fenómeno aportando elementos técnicos para orientar y proponer las acciones de intervención en las unidades y elementos conexos en captación, canal de aducción y desarenador.

\section{MATERIALES Y MÉTODOS}

\section{Zona de estudio}

El municipio de Calarcá se sitúa al oriente del departamento del Quindío, en el flanco occidental de la cordillera Central en una altitud media de 1.703 msnm, su topografía es quebrada y abrupta, con pendientes fuertes (1). En este municipio se tiene, en la zona urbana 95 barrios abastecidos por un sistema de acueducto administrado por la empresa MULTIPROPÓSITO DE CALARCÁS.A. ESP.

El agua es tomada mediante cuatro bocatomas que se ubican en el río Santo Domingo, Qda. El Salado, Qda. San Rafael y Qda. El Naranjal. La Cuenca Hidrográfica del río Santo Domingo, principal fuente de abastecimiento de agua del municipio, limita geográficamente con la cuenca del río Navarco al norte, con el departamento del Tolima al oriente, con la cuenca del río Verde al sur y con la cuenca del río Quindío al occidente. El río Santo Domingo corre inicialmente en dirección sur - norte, recibe las aguas de las quebradas El Campanario, Uritá, Planadas, San Antonio, La Gata, El Oso, San Rafael y luego de un recorrido cercano a los $12 \mathrm{Km}$., recibe las aguas de la Quebrada el salado. En la Tabla 1 se identificaron los parámetros morfométricos para la cuenca del río Santo Domingo y el Salado.

El estudio se desarrolló en los sitios de captación ubicados en la Qda. El Salado y el río Santo Domingo en la zona alta y encañonada de la cuenca. Las estructuras de captación se integran en una misma aducción y

Tabla 1. Parámetros morfométricos de las cuencas en estudio

\begin{tabular}{ccccccc}
\hline Cuenca & $\begin{array}{c}\text { Cota } \\
\text { Nacimiento } \\
\text { msnm }\end{array}$ & $\begin{array}{c}\text { Cota } \\
\text { Desemocadura } \\
\text { msnm }\end{array}$ & $\begin{array}{c}\text { Longitud } \\
\text { Río } \\
\mathrm{km}\end{array}$ & $\begin{array}{c}\text { Pendiente } \\
\text { Río } \\
\%\end{array}$ & $\begin{array}{c}\text { Pendiente } \\
\text { Cuenca } \\
\%\end{array}$ & $\begin{array}{c}\text { Área } \\
\mathrm{km}^{2}\end{array}$ \\
\hline S alado & 2300 & 1450 & 3.645 & 23.32 & 53.08 & 4.497 \\
S. Domingo & 3590 & 1450 & 12.821 & 16.69 & 54.33 & 56.399 \\
\hline
\end{tabular}


desarenador, denominándose sistema de captación Santo Domingo - Salado.

En la Figura 1, se observa aguas arriba de la captación del rio Santo Domingo, un depósito de bloques de roca de diferentes tamaños. La dinámica hidráulica de la fuente y las altas pendientes, permiten flujo continuo de materiales que ha hecho que la estructura, una bocatoma de fondo inclinada, presente dificultades

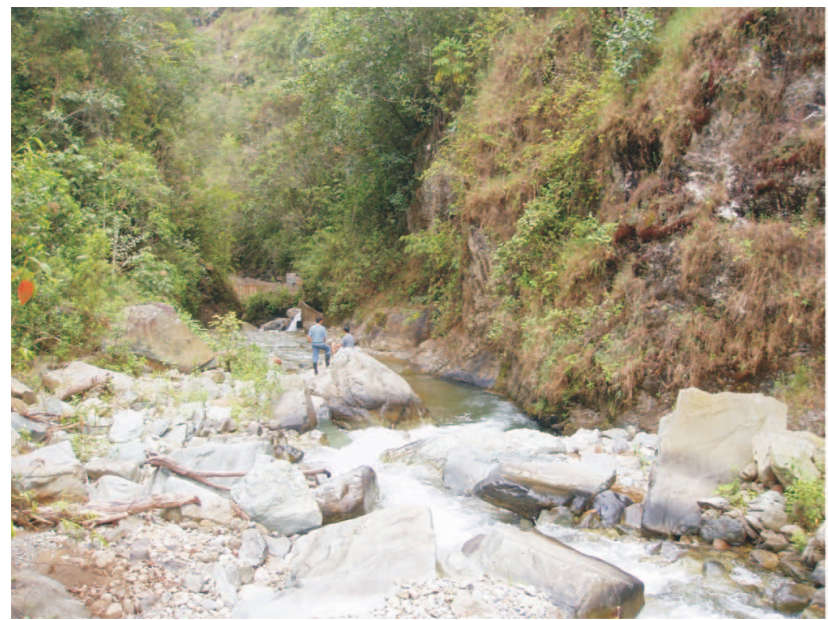

estructurales y operativas por recibir directamente impactos del flujo del material circulante en el cauce derivado de la energía generada cuando se presentan avenidas en la cuenca. Esta situación compromete el funcionamiento y la operatividad de las bocatomas, unidades de caja de recolección, canal de aducción, regulación, control de caudal y desarenador. El mayor impacto sobre la estructura fue la avalancha del 24 de diciembre de 1999.

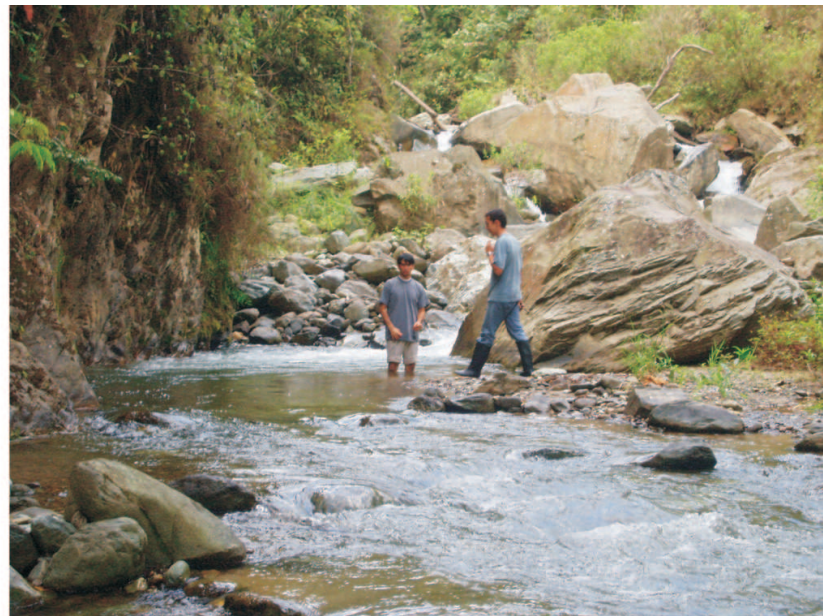

Figura 1. Zona de captación Río Santo Domingo

\section{Recolección de información}

Llevar a cabo el estudio, demandó en primera instancia, una amplia consecución de información referente a cartografía, registros de caudal, precipitación en la zona y caudales concedidos; de igual manera, se efectuó un levantamiento de información para establecer las condiciones de funcionamiento y operación, como también el comportamiento de las unidades que integran el sistema Santo Domingo - Salado; la recolección de información continuó revisando la literatura especifica de diseños de acueductos en el país.

La estación seleccionada para tomar registros de precipitación fue la estación Planadas, ubicada en la cuenca a $2.350 \mathrm{msnm}$. La recolección de información en campo inició con el levantamiento topográfico de la zona de captación y cada una de las unidades que integran el sistema Santo Domingo - El Salado. Se efectuaron aforos en el canal de aducción, con el fin de establecer las variables hidráulicas y el comportamiento del caudal con las unidades de control y regulación existente.

\section{Procesamiento de la información}

Estudio Hidrológico: Para la estimación de caudales medios se usó la metodología del Balance Hidrológico a largo plazo, obteniendo un caudal promedio de $0.16 \mathrm{~m}^{3} / \mathrm{s}$ para la quebrada $\mathrm{EI}$ Salado y $1.95 \mathrm{~m}^{3} / \mathrm{s}$ para el Río Santo Domingo. Para el cálculo de los caudales máximos y mínimos se empleó el análisis de frecuencia asociado a diferentes periodos de retorno. En la Tabla 2 se presenta el resumen de los caudales para cada una de las cuencas en estudio.

Evaluación Hidráulica del Sistema: Mediante información primaria y secundaria se evaluó el sistema Santo Domingo - El Salado desde la zona de captación hasta el desarenador, encontrándose lo siguiente: 
Monsalve D., Elkin A., García R., Pedro L., Lozano S., Gabriel - 133

Tabla 2. Resumen de caudales en las cuencas en estudio

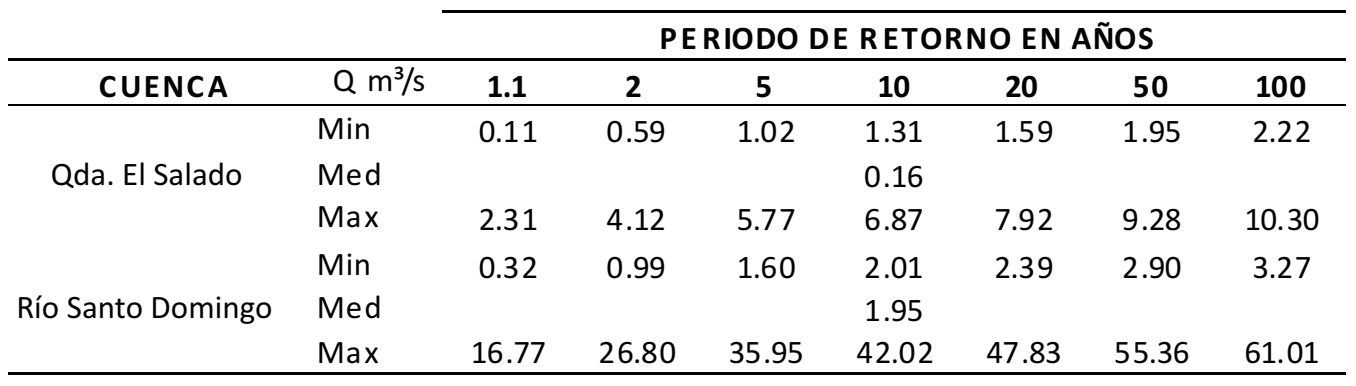

- Caudales Concedidos: El caudal concedido por la autoridad ambiental mediante la Resolución $N^{\circ} 0392$ del 1 de abril de 2004, es de 220 l/s, el cual se encuentra distribuido en la siguiente forma: Río Santo Domingo (75 l/s), Qda. El Salado (70 l/s), Qda. San Rafael (60 l/s) y Qda. Naranjal (15 l/s). Esta disposición fortalece la operación del sistema, dado que la combinación para los niveles de captación permite el suministro desde diferentes fuentes. Sin embargo, la concesión limita la posibilidad de captar volúmenes mayores en caso de situaciones extraordinarias, como por ejemplo el detrimento de la calidad del agua por amenazas generadas desde la vía Calarcá Cajamarca.

- Zona de Captación: En el río Santo Domingo se analizó la zona de entrada a la captación, ya que se deposita gran cantidad de material y existe permanente transito de elementos gruesos que hacen vulnerable la estructura de captación. Analizada la situación se planteo establecer una estructura para proteger la bocatoma y la caja de derivación de caudal, garantizando el libre tránsito del material transportado por el río.

- Estructura de Captación: Para evaluar las bocatomas existentes y proceder con el diseño definitivo de la captación sobre el río Santo Domingo, se consideraron los parámetros expuestos en el título B de la norma RAS (2), y en bibliografía específica del tema (3-6).
- En la bocatoma El Salado no se observó ningún tipo de alteración, la estructura se encontraba intacta, no se evidenciaban indicios de flujo de material que pudiera influir en la vida de la estructura. Aguas abajo de la estructura, por la caída del agua se pueden llegar a presentar problemas de socavación sobre la base de la estructura. La reja de toma presenta una separación entre barrotes muy variable.

- Con base en los datos tomados en campo se efectuó el chequeo de la reja para establecer la capacidad de captación de la estructura. Se encontró que bajo las condiciones de área neta de reja: $1.86 \mathrm{~m}^{2}$ y caudal de captación limitado por la concesión Q: $0.07 \mathrm{~m}^{3} / \mathrm{s}$, se obtiene una altura de lámina de agua $\mathrm{H}$ : 0.2 milímetros, estableciéndose que la toma esta sobrediseñada y que el caudal que ingresa al sistema es superior al demandado, por lo que en la aducción se hace necesario evacuar los excesos al río.

- En la bocatoma del río Santo Domingo se encontró grandes variaciones de caudal entre los periodos de estiaje y de crecientes máximas. En el sistema de captación no existía reja, ni elementos para el control del caudal captado; además, aguas abajo de la toma la caída del agua estaba socavando la estructura. La Figura 2 ilustra los daños ocasionados por el constante flujo de rocas en la bocatoma de Santo Domingo. 


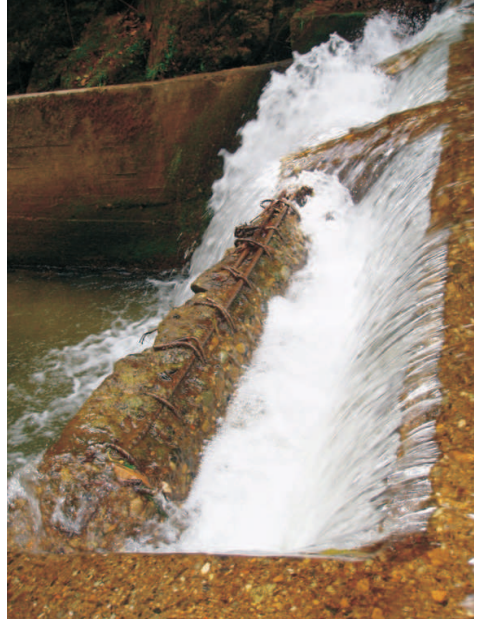

PERDIDA DE LA REJA

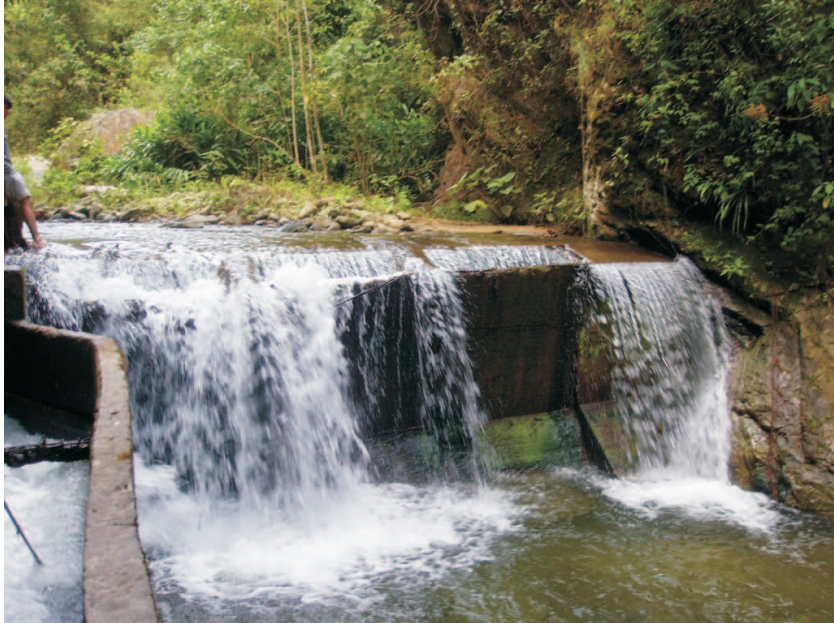

SOCAVACIÓN DE LA ESTRUCTURA

Figura 2. Alteración en la estructura de captación Santo Domingo

De los elementos que teóricamente integran este tipo de bocatoma, no se encontraron la reja, los muros de protección del tránsito de crecientes, y se encontró en mal estado el canal y la caja de recolección.

- Canal de Aducción: En su recorrido el canal presenta dos zonas (Figura 3); la primera desde la cámara de salida de la Bocatoma Santo Domingo hasta la intersección del canal de derivación de la Bocatoma El Salado, con un desarrollo de $47.74 \mathrm{~m}$ y una pendiente promedio del $1.58 \%$. Exhibe un comportamiento hidráulico adecuado con desarrollo de velocidades altas (> $2.97 \mathrm{~m} / \mathrm{s})$, controlando con ello la deposición de sedimentos. En la abscisa 29.30 se presenta una compuerta lateral controlada por la que se

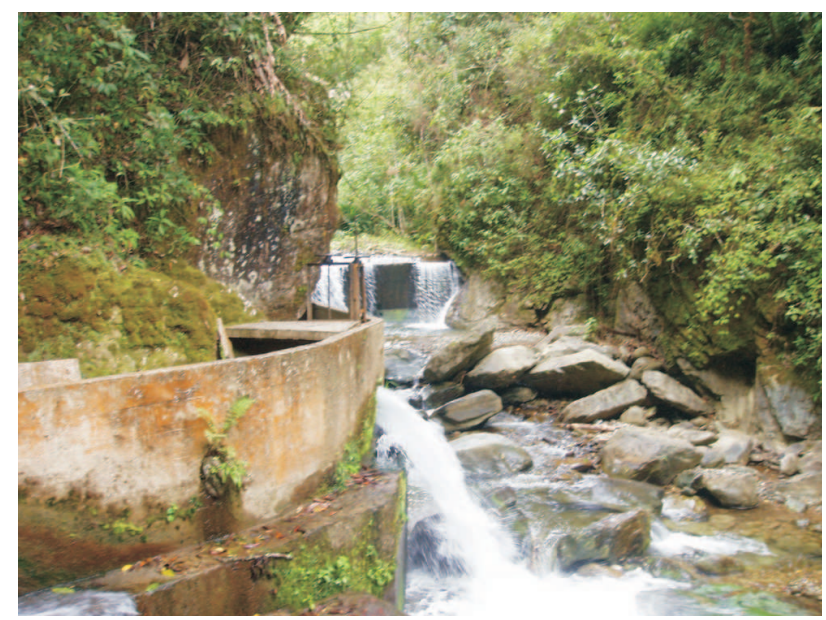

Figura 3. Zonas de aducción sistema Santo Domingo - El Salado vierte los excesos captados, aunque las condiciones de operación hacen de ella una estructura ineficiente para registrar el caudal neto derivado hacia el desarenador. Al final del tramo, confluyen las aguas captadas en la Qda. El Salado, las cuales se controlan con una compuerta que opera adecuadamente (7).

- La segunda zona va desde la confluencia de la Bocatoma El Salado hasta el Desarenador, con un desarrollo de $21.03 \mathrm{~m}$ y una pendiente media $6.02 \%$. En su tramo final descargaba el agua con una pendiente del $48.36 \%$ generando altas cabezas de velocidad y turbulencia que no lograban disiparse con las obras proyectadas aguas abajo y restringían la operación del desarenador.

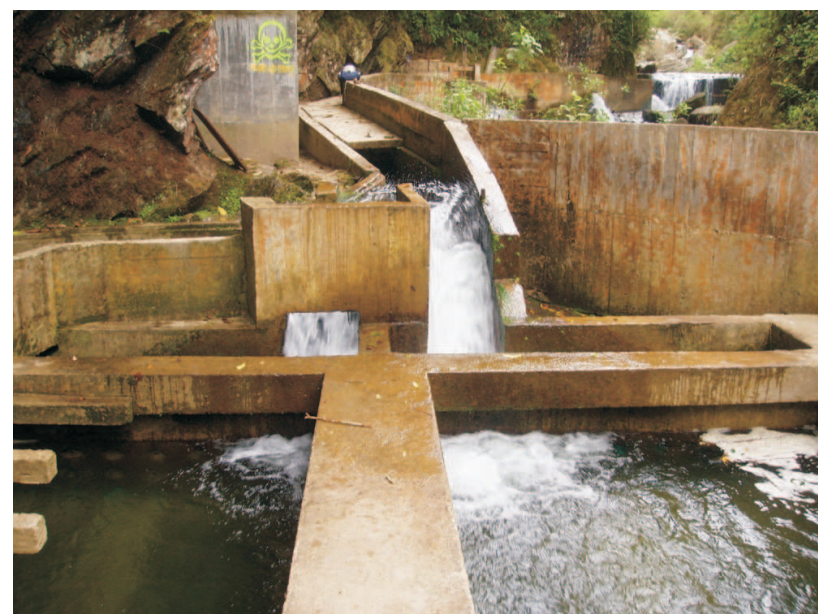

Rev. Invest. Univ. Quindío (21): 130-139. Armenia-Colombia 
- Si desde el río Santo Domingo se derivan $75 \mathrm{I} / \mathrm{s}$ y desde la Qda. El Salado se deriva 70 l/s, el caudal de diseño para la primera zona debería ser $75 \mathrm{l} / \mathrm{s}$, mientras que para la segunda zona se tiene $145 \mathrm{l} / \mathrm{s}$ (en su límite máximo). Si el sistema de captación El Salado sale de funcionamiento, la primera zona podría conducir los 145 l/s. En visita de campo, registrando velocidades superficiales en la primera zona, se cálculo un caudal de $520 \mathrm{l} / \mathrm{s}$ para la sección típica identificada (Figura 3). Queda claro que no existe un control de caudal en la bocatoma ni en el canal, y a través del vertedero lateral se estaría evacuando el $72 \%$ del caudal captado.

- Desarenador: Es de tipo convencional de dos unidades, cada una está constituida por cuatro

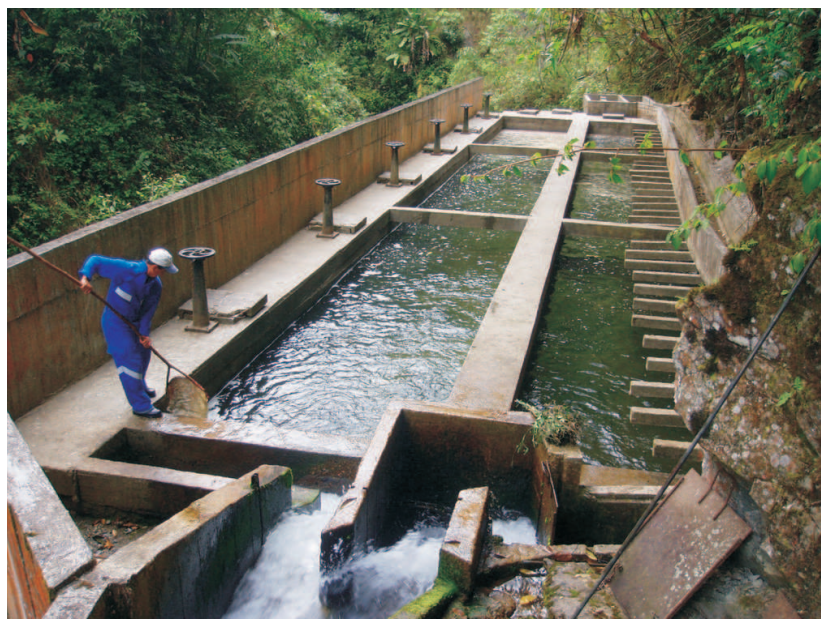

zonas; entrada, sedimentación, almacenamiento de lodos y salida, y cuyas dimensiones principales son: 26.4 metros de longitud, 2.85 metros de ancho y 1.55 metros en promedio de profundidad.

- Se identificó en la zona de transición entre el canal de aducción y la entrada al desarenador la no existencia de una estructura de disipación de energía, aquietamiento y distribución de caudal; la alta pendiente del canal al llegar al desarenador hace que el agua impacte contra la pantalla deflectora generando que el flujo en el desarenador, considerado teóricamente laminar, se convierta en turbulento y se presenten zonas de corto circuito que hacen poco eficiente la zona de sedimentación (Figura 4).

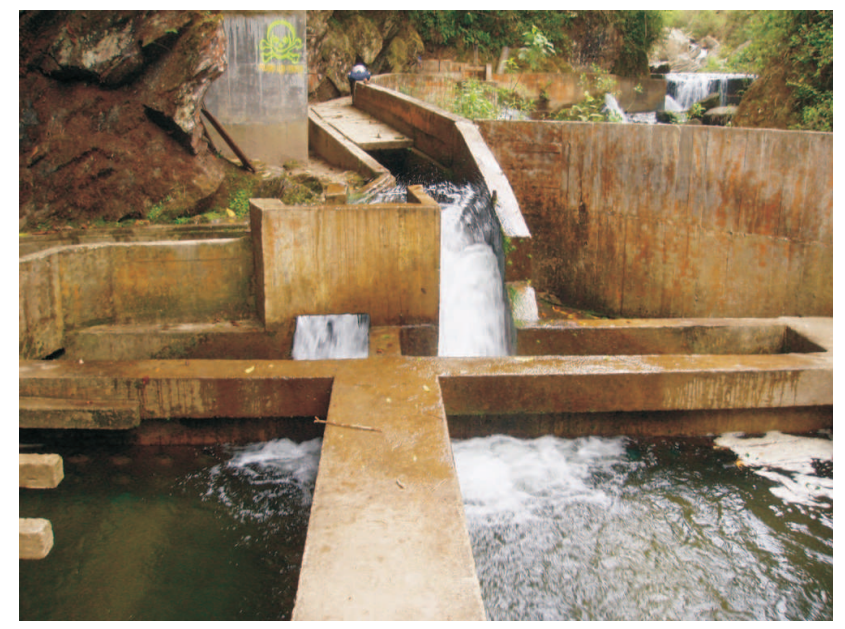

Figura 4. Desarenador y zona de entrada

\section{RESULTADOS Y DISCUSIÓN}

Siendo $220 \mathrm{l} / \mathrm{s}$ el caudal total concedido por la Corporación Autónoma Regional del Quindío a la empresa MULTIPROPOSITOS DE CALARCA SA. ESP, distribuido en cuatro fuentes de abastecimiento, puede presentarse el caso de que por algún tipo de contingencia, los $70 \mathrm{l} / \mathrm{s}$ demandados de la Qda El Salado se tengan que extraer del río Santo Domingo, por lo cual la toma en Santo Domingo se proyecta para captar $145 \mathrm{I} / \mathrm{s}$.

\section{Zona de captación}

Del análisis de la situación presente en la zona de captación de Santo Domingo, se estableció que cualquier tipo de alternativa a emplear para captar agua, estará condicionada en funcionamiento y operación por el comportamiento de la corriente aguas arriba; por tal motivo, se efectuó una intervención de tal manera que no se interfiera con las condiciones naturales y la dinámica del río. 
Se propuso mejorar y proteger el cauce donde se proyecta ubicar la captación, mediante una estructura cuyo objetivo primordial es evacuar de manera rápida y segura todo aquel material o escombro de gran tamaño que entre en el cauce del río y que pueda llegar a depositarse sobre la captación.

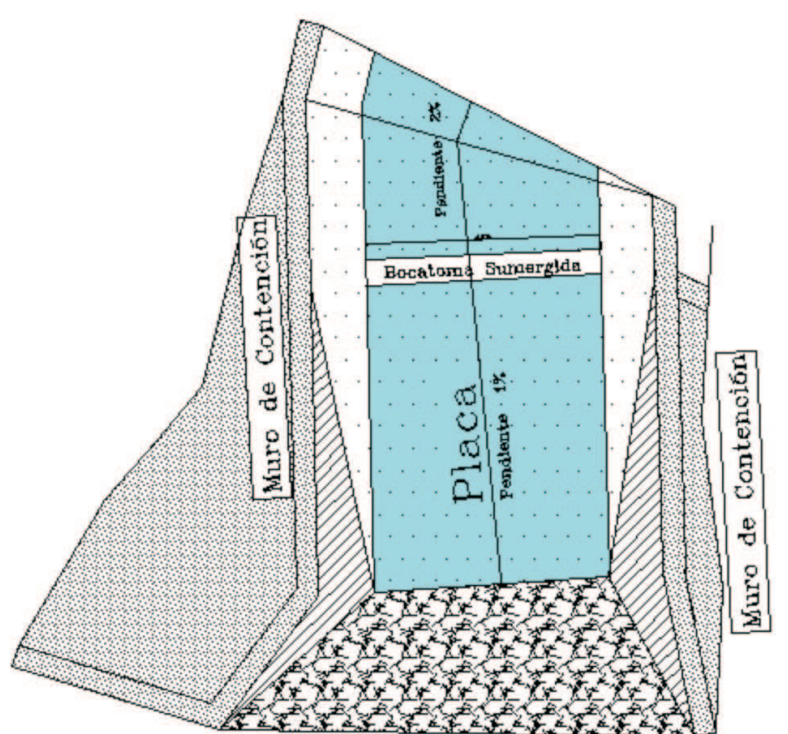

La solución concebida como se observa en la Figura 5, es una estructura de evacuación constituida por una sección a la entrada que permite el tránsito y evita el depósito de material. La capacidad hidráulica calculada en la sección permite evacuar un caudal máximo de $47.83 \mathrm{~m} 3 / \mathrm{s}$ para un periodo de retorno de 20 años y un tirante de $1.2 \mathrm{~m}$.

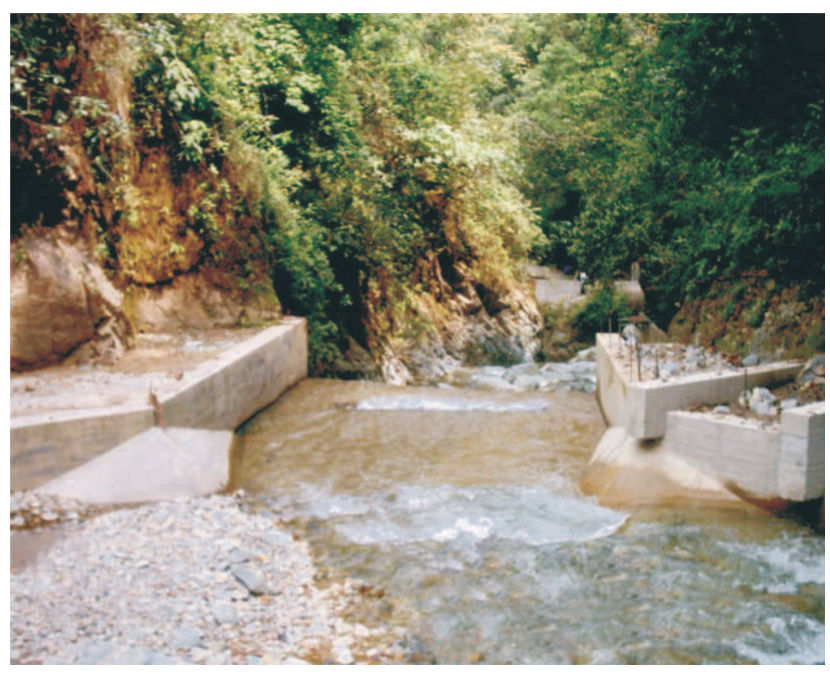

rigura s. tstructura de evacuación de material

\section{Estructura de captación}

La bocatoma diseñada y recomendada según las características de la zona, es una bocatoma de tipo sumergida o de fondo para caudales mayores, la cual mediante una rejilla encausa el flujo y lo dirige al canal de aducción que conecta con la caja de recolección de caudales hasta entregarlo al canal de aducción. Mediante la zona de entrada de la estructura se contiene y conduce el flujo de material permitiendo la captación (Figura 6).

Cuando se plantea la toma, se define un área neta de captación para el caudal de diseño, que a su vez depende de una altura de lámina de agua sobre la rejilla. Esta condición no es permanente debido a que en la fuente transitan diferentes caudales y se presenta variación en la lámina de agua sobre la estructura de captación; el caudal de diseño, se convierte entonces en un valor teórico e ideal que se presenta esporádicamente durante el tiempo de funcionamiento del sistema. Como no se puede graduar constantemente el área de captación de acuerdo con la lámina de agua presente, por resultar costoso, inoperante y poco funcional, el caudal debe ser regulado aguas abajo hasta obtener el volumen requerido para el desarenador.

En esta situación tan particular donde la fuente por su dinámica y la variación permanente de caudales, hace que el caudal captado sea superior al de diseño, no es practico proyectar la típica caja de recolección de caudales como se referencia en los textos guía en Colombia de (4-6); por lo tanto, se proyectó una estructura o caja para permitir el paso de caudal desde el canal de recolección en la bocatoma hasta el canal de conducción. La bibliografía hace referencia además que en esta unidad se debe hacer el control del caudal de excesos, lo que no es posible en esta bocatoma por las condiciones hidráulicas presentes al entrar el agua a la caja. La regulación del caudal se plantea hacerse aguas abajo en el canal de aducción. 

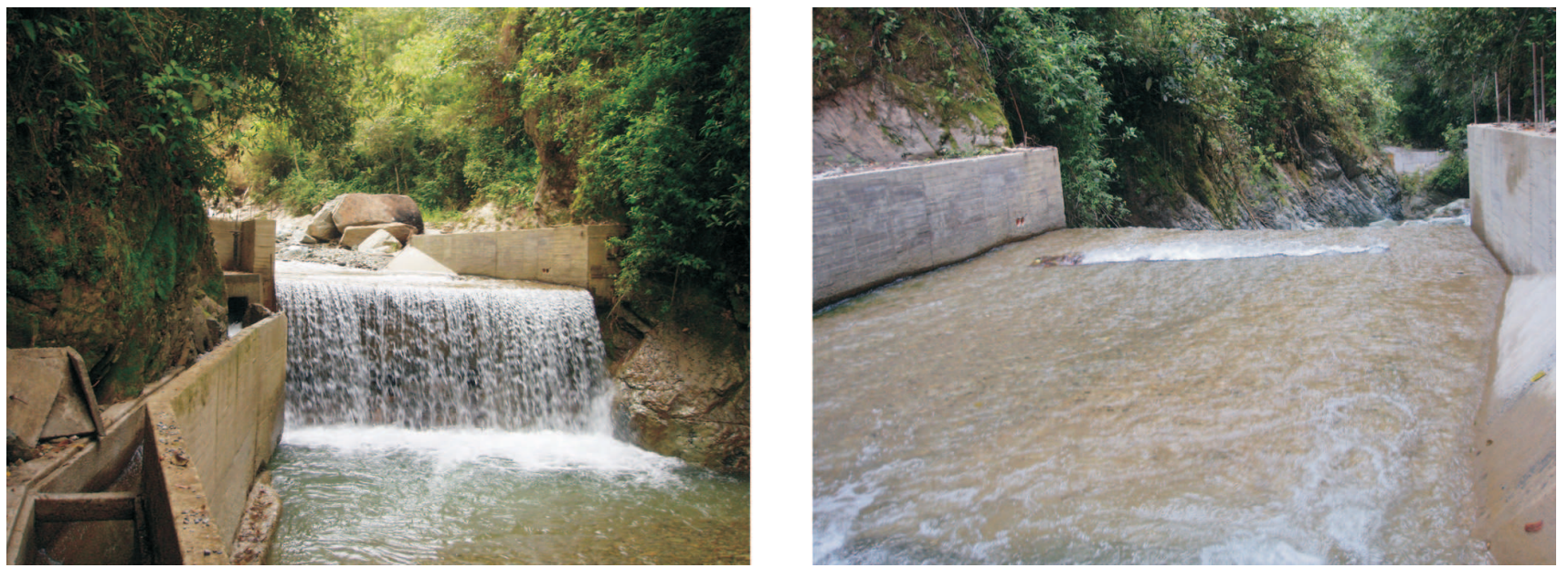

Figura 6. Bocatoma de fondo proyectada

\section{Canal de aducción}

En esta unidad se presentaban altas velocidades, no existían medios para registrar el caudal captado, ni elementos de regulación y aliviadero del caudal de excesos. El estudio en el canal se efectuó con base en dos condiciones: (1) Estará en capacidad de conducir un caudal de $145 \mathrm{l} / \mathrm{s}$ y, (2) deberá existir una estructura de control que permita controlar y registrar los caudales enviados.

Para conocer el comportamiento del tramo, se llevo a cabo una modelación en HEC-RAS considerando un vertedero lateral controlado por una compuerta deslizante.
Se estableció que el canal tiene la capacidad para conducir caudales superiores a $520 \mathrm{l} / \mathrm{s}$. De hecho, puede transportar $980 \mathrm{l} / \mathrm{s}$ generando una evacuación máxima en el vertedero de $410 \mathrm{l} / \mathrm{s}$. Es decir, al desarenador llegaría más agua de la necesaria, obligando a disponer de controles de caudal. Se presentan también velocidades superiores a $2.00 \mathrm{~m} / \mathrm{s}$, por lo que la sedimentación no será un problema. Luego de pasar la estructura de control, para cualquier condición de flujo la velocidad tiende a incrementarse, por lo que aguas abajo se debió disipar la energía.

La estructura está compuesta por un vertedero rectangular de pared gruesa y una compuerta deslizante con orificio de descarga que fue proyectada con el fin de garantizar la llegada del caudal máximo de $145 \mathrm{l} / \mathrm{s}$ al desarenador. (Figura 7).

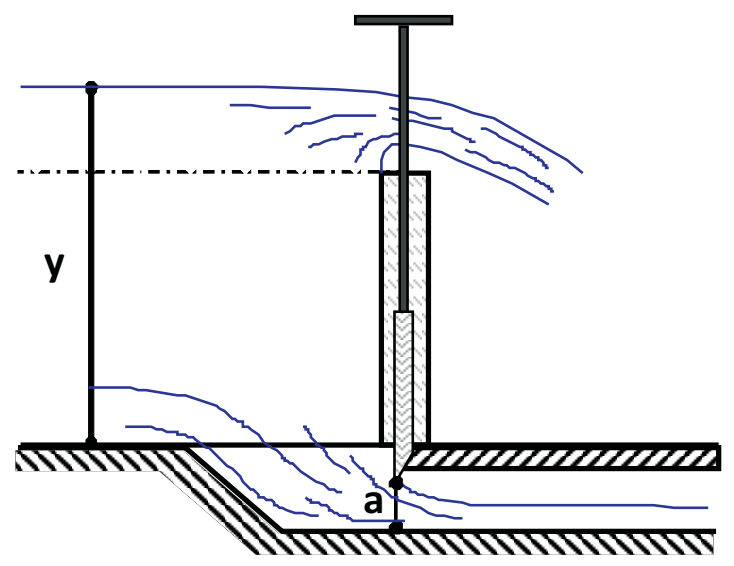

Figura 7. Estructura para control de flujo Zona 1

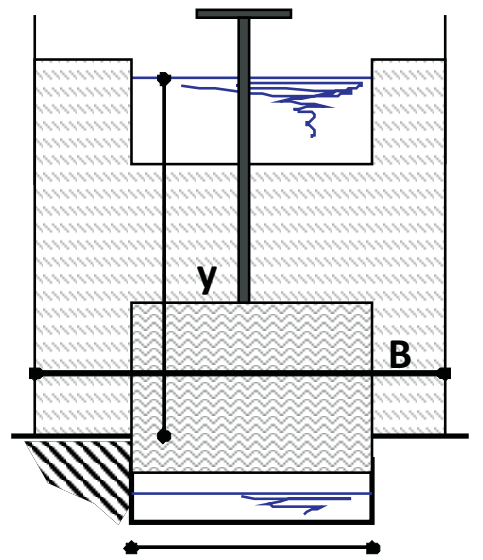

b

Rev. Invest. Univ. Quindío (21): 130-139. Armenia - Colombia 
Como la pendiente del canal antes de llegar al desarenador ocasionaba alta velocidad del agua y alteraba la entrada del desarenador, se propuso la construcción de escalas disipadoras a partir del cambio de pendiente, K0+54.87 (Figura 8). El diseño hidráulico estuvo limitado al cálculo de una estructura escalonada en condiciones de flujo crítico.

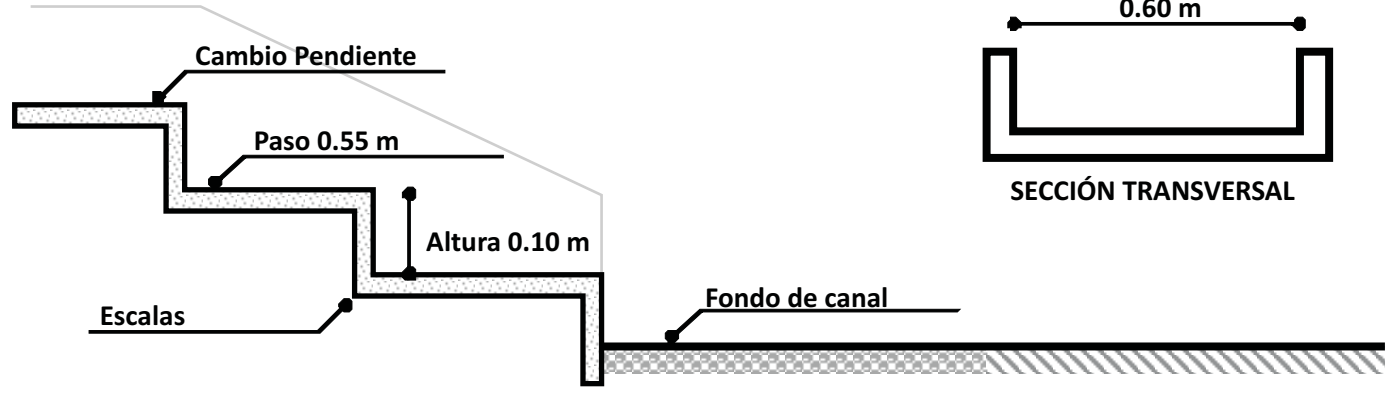

Figura 8. Detalle escala disipadora

\section{Desarenador}

No se encontraron inconvenientes en la zona de lodos y salida de caudal. En cuanto a la zona de sedimentación para determinar la eficiencia en la remoción de partículas, se realizó un análisis basado en la teoría de sedimentación, la Ley de Stokes y el Número de Hazen (8), contemplando $75 \mathrm{l} / \mathrm{s}$ y $145 \mathrm{l} / \mathrm{s}$ como posibles caudales de entrada. El desarenador presento un funcionamiento aceptable cuando ingresan caudales altos y un funcionamiento optimo cuando los caudales son inferiores.

Como la principal dificultad se presentaba en la zona de entrada del desarenador, donde el canal se repartía desigualmente e impactaba con las pantallas deflectoras generando flujo turbulento, se determino construir una estructura de aquietamiento y distribución de caudal para garantizar en cada unidad el caudal necesario.

En la Figura 9 se observa la estructura de aquietamiento y la distribución de caudal mediante vertederos a cada una de las unidades del desarenador. El máximo caudal que llega al desarenador es $145 \mathrm{l} / \mathrm{s}$, ya sea porque están simultáneamente en funcionamiento El Salado (70 l/s) y Santo Domingo (75 l/s), o porque solo se está captando de Santo Domingo (145 I/s). Los 145 $\mathrm{l} / \mathrm{s}$ que llegaran al desarenador deberán, en caso de que se cierre una unidad, pasar por uno de los vertederos y dirigirse a la unidad en funcionamiento.

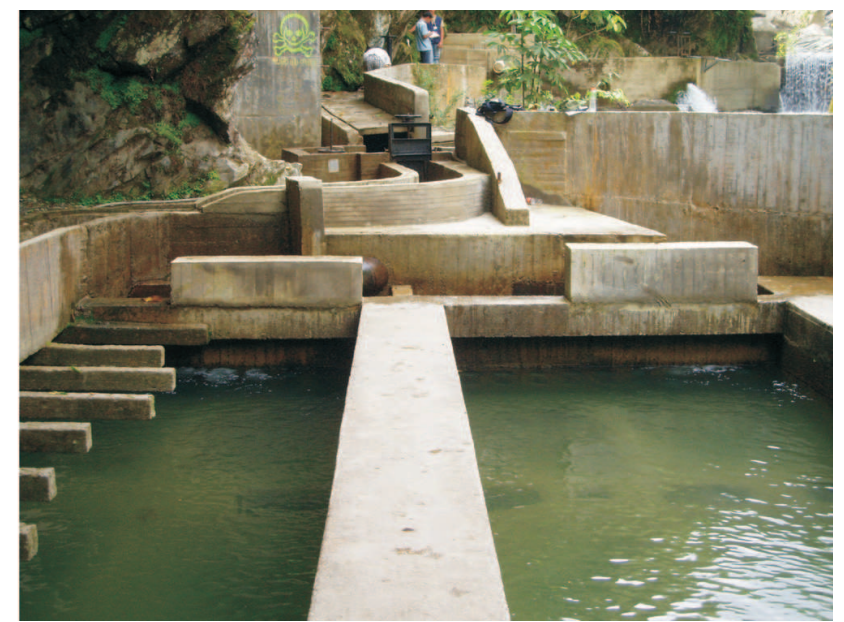

Figura 9. Estructura de distribución de caudal y entrada al desarenador 
Monsalve D., Elkin A., García R., Pedro L., Lozano S., Gabriel - 139 -

\section{CONCLUSIONES}

La bibliografía referente al diseño y optimización de sistemas de abastecimiento de agua en Colombia es reducida, sus contenidos, aunque recogen los parámetros necesarios de la hidráulica y los lineamientos de las diversas normas para realizar diseños, cálculos y proyecciones se han convertido en muchos casos en una receta que se aplica sin el debido análisis y evaluación de las condiciones presentes en el sitio donde se desea desarrollar un proyecto de abastecimiento de agua.

En el río Santo Domingo, una fuente de alta montaña con condiciones tan particulares respecto al comportamiento hidrológico e hidráulico en la zona, y donde el sistema de abastecimiento existente ha sufrido problemas saliendo de funcionamiento y dejando al municipio sin agua, no se permitió la aplicación expresa de los parámetros típicos citados en la bibliografía; se hizo necesario afrontar el estudio desde dos fases: Fase 1. Analizar en detalle la situación en la zona de captación, con el fin de obtener una radiografía del comportamiento del río y las unidades dispuestas en la zona. Fase 2. Realizar el diseño y cálculo de cada una de las unidades de acuerdo con parámetros hidráulicos y condicionados al comportamiento natural de la fuente, cuya finalidad fue la mínima interferencia del sistema natural.

\section{BIBLIOGRAFÍA}

1. Calarcá. Plan Básico de Ordenamiento Territorial. Municipio de Calarcá. 2000-2007.

2. RAS, 2000. Reglamento Técnico del sector de agua potable y saneamiento básico Ministerio de Desarrollo. 2000.

3. Materon, H. Obras Hidráulicas Rurales. Universidad del Valle. 1997

4. López C. R. Elementos de Diseño para acueductos y alcantarillados. Escuela Colombiana de Ingeniería. Segunda edición. 2006.

5. Corcho Fy Duque J. Acueductos Teoría y diseño. Universidad de Medellín.

6. Pérez P. Jorge. Acueductos y Alcantarillados. Primera edición 1995.

7. Chow, V. T., et al. Hidrología Aplicada. Mc GRAW-HILL. Santafé de Bogotá. 1994.

8. Arboleda, J. Teoría y práctica de la purificación del agua, tercera edición. Mc GRAW-HILL INTERAMERICANA, S.A. Santafé de Bogotá. 2000. 